\title{
Flexible and wearable wire-shaped microsupercapacitors \\ based on highly aligned titania and carbon nanotubes
}

\author{
Tao Chen, Liming Dai*
}

Center of Advanced Science and Engineering for Carbon (Case4Carbon), Department of Macromolecular Science and Engineering, Case Western Reserve University, 10900 Euclid Avenue, Cleveland, OH 44106 (USA).

* Corresponding author.

E-mail address: liming.dai@case.edu

\begin{abstract}
Wire-shaped devices, such as solar cells and supercapacitors, have attracted great attentions due to their unique structure and promise to be integrated into textiles as portable energy source. To date, most reported wire-shaped supercapacitors were developed based on carbon nanomaterial-derived fiber electrodes whereas titania was much less used, though with excellent pseudocapacitvie properties. In this work, we used a titanium wire sheathed with radially aligned titania nanotubes as one of the electrodes to construct all-solid-state microsupercapacitors, in which the second electrode was carbon nanotube fiber or sheet. The capacitance of the resulting microsupercapacitor with a CNT sheet electrode $\left(1.84 \mathrm{mF} \mathrm{cm}^{-2}\right)$ is about three time of that for the corresponding device with the second electrode based on a single CNT yarn. The unique wire-shaped structure makes it possible for the wire-shaped microsupercapacitors to be woven into various textiles and connected in series or parallel to meet a large variety of specific energy demands.
\end{abstract}

Keywords: microsupercapacitor, wire-shaped, wearable, carbon nanotube, titania

\section{Introduction}

The wearable wire-shaped electronics for energy conversion and/or storage have recently attracted considerable attention, and have great potentials for various applications, especially as integrated electronics with clothing and other textiles.[1-3] In particular, wire-shaped energy conversion devices converting light,[4,5] mechanical [6] and/or heat [7] into electricity have been widely investigated. In order to utilize the electricity thus generated more efficiently and to effectively extend the 
duration of its usage, wearable wire-shaped energy storage devices matching with those wire-shaped energy conversion counterparts are needed. Apart from the potential use as electronics with clothing and other textiles, wire-shaped energy storage devices (e.g., microsupercapacitors) can also provide electrical energy for other electronics, including on-body sensors.[8-13] Microsupercapacitors (also known as electrochemical capacitors at microscale) possess high power density and reliability,[14] which can satisfy the power requirements of various wearable electronics. Of particular importance to their practical applications, microsupercapacitors with high efficiencies can be fabricated in the form of all-solid-state devices. In this context, it is highly desirable to develop high-performance, lightweight, flexible and wire-shaped microsupercapacitors for powering wearable electronics.

Wire-shaped (micro)supercapacitors have similar structure with their planar counterparts, including two electrodes, electrolyte and a separator.[8-13] Most wire-shaped solid (micro)supercapacitors reported to date were constructed by using nickel wire, carbon nanotube wire, or plastic wire as the wire-shaped electrode and a polymer gel as the electrolyte,[8-13] though their performance has not reached to the level as expected - probably due to the limited surface area for metal wires or the limited electrical conductivity intrinsically associated with plastic wires. Titanium dioxide $\left(\mathrm{TiO}_{2}\right)$ has been used as the electrode for supercapacitors to achieve high specific capacitance as well as high energy and power densities.[15-17] Highly aligned $\mathrm{TiO}_{2}$ nanotube arrays with a high specific area can be easily obtained by electrochemical anodization and are attractive as electrode materials in microsupercapacitors.[15-17] On the other hand, carbon nanomaterials (e.g., carbon nanotubes, graphene) have been widely used as active electrodes in microsupercapacitors with high performance due to their high surface area and high conductivity.[18-26]

Herein, we report the first flexible and wearable wire-shaped microsupercapacitor based on a wire-shaped microelectrode made from a titanium (Ti) wire sheathed with radially aligned titania nanotubes, in which the radially aligned semiconducting titania nanotubes can not only increase the surface area of the electrode but also act as a separator between the Ti fiber electrode and the outmost carbon nanotube (CNT) yarn or sheet electrode, which was twisted around the outer surface of the anodized titanium (Ti) wire (Fig. 1). 


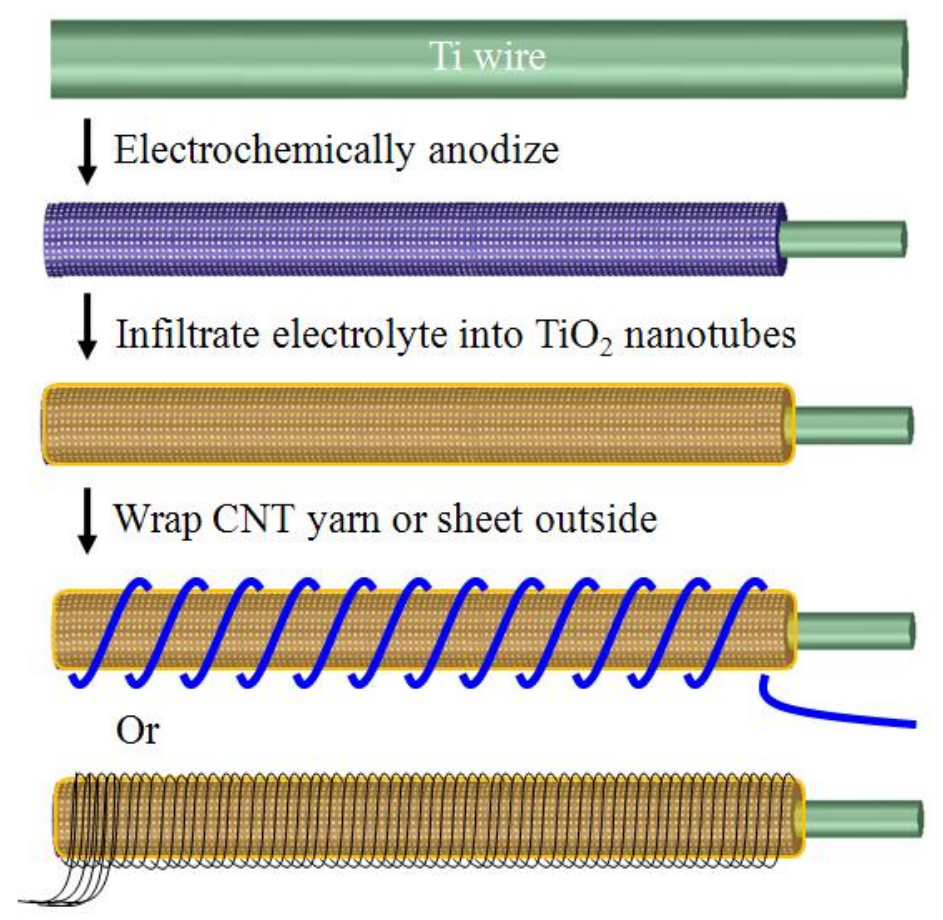

Fig. 1. Schematic illumination of procedures for constructing the wire-shaped microsupercapacitors based on a wire-shaped electrode made from a titanium (Ti) wire sheathed with radially aligned titania nanotubes and wrapped with highly-aligned carbon nanotube yarn or sheet.

\section{Materials and Methods}

The vertically-aligned CNT array was synthesized by chemical vapor deposition using ethylene as the carbon source $(120 \mathrm{sccm})$, the sputter-coated $\mathrm{Fe}(1.2 \mathrm{~nm}) / \mathrm{Al}_{2} \mathrm{O}_{3}$ ( $3 \mathrm{~nm}$ ) catalyst on a silicon wafer as the nanotube growth substrate and a mixture of argon and hydrogen (flow rate of $400 \mathrm{sccm} / 30 \mathrm{sccm}$ ) as the carrier gas at 750 ${ }^{\circ} \mathrm{C}$.[27,28] The resultant-vertically aligned CNT array was then directly drawn into horizontally-aligned CNT sheet or spun into yarns using a microprobe with rotating rapid of $2000 \mathrm{rpm}$. Aligned titania nanotube array was radially grown on the surface of a Ti wire (purchase from Alfa Aesar) with a diameter of $127 \mu \mathrm{m}$ through an electrochemical anodic oxidation method in the electrolyte solution containing 0.3 wt $\% \mathrm{NH}_{4} \mathrm{~F}$ (Sigmaadrich) and $2 \mathrm{vol} \% \mathrm{H}_{2} \mathrm{O}$ in ethylene glycol (Sigmaadrich) at $60 \mathrm{~V}$ for 2 h.[29] The growth was carried out in a two-electrode electrochemical cell using $\mathrm{Ti}$ wire as the working electrode and platinum plate as the counter electrode. The anodized $\mathrm{Ti}$ wires were washed with deionized water to remove the residual electrolyte, followed by annealing at $500{ }^{\circ} \mathrm{C}$ in air for $1 \mathrm{~h}$. 
The wire-shaped microsupercapacitors were fabricated as follows. The annealing anodized Ti wire was immersed into a gel electrolyte solution of poly(vinyl alcohol) powder (10 g, Sigmaadrich) and $\mathrm{H}_{3} \mathrm{PO}_{4}(10 \mathrm{~g}$, Sigmaadrich) in water (100 mL) for 10 min, followed by evaporating the water in air. Then, the CNT yarn was carefully wrapped around the above sample by hand. In the case of CNT sheet electrode, the CNT sheet was drawn from a vertically-aligned CNT array onto the surface of the electrolyte-coated anodized $\mathrm{Ti}$ wire by rotating the $\mathrm{Ti}$ wire to wrap the CNT sheet from one end to another end of the Ti wire. Additional electrolyte was coated onto the assembled devices to enhance the contact state between the CNT yarns or sheet with the electrolyte. Finally, both electrodes of CNT yarns or sheet and Ti wire were connected to external circuit using silver paste for subsequent characterization. The carbon nanotube structure was characterized by SEM (JEOL JSM-6510LV/LGS operated at $20 \mathrm{kV}$ ). Electrochemical measurements were performed on an electrochemical working station (CHI 760C, U.S.A.).

\section{Results and discussion}

As schematically shown in Fig. 1, the titania nanotube array was prepared by electrochemical anodizing a Ti wire as a working electrode in the electrolyte solution containing $0.3 \mathrm{wt} \% \mathrm{NH}_{4} \mathrm{~F}$ and $2 \mathrm{vol} \% \mathrm{H}_{2} \mathrm{O}$ in ethylene glycol for 2 hours at a voltage of $60 \mathrm{~V}$ by using a platinum plate as the counter electrode. The anodized Ti wire was then washed with deionized water, followed by annealing at $500{ }^{\circ} \mathrm{C}$ for 1 hour. Thereafter, the resultant anodized Ti wire was immersed into a gel electrolyte solution of poly(vinyl alcohol) powder $(10 \mathrm{~g})$ and $\mathrm{H}_{3} \mathrm{PO}_{4}(10 \mathrm{~g})$ in water $(100 \mathrm{~mL})$ for $10 \mathrm{~min}$, followed by evaporating the water in air. Highly aligned CNT yarn or sheet was wrapped around the air-dried hybrid wire, and the infiltrated gel electrolyte can effectively prevent the outer CNT electrode from contacting with the core $\mathrm{Ti}$ wire. Finally, the opposite ends of the $\mathrm{Ti}$ wire and CNT yarn or sheet wrapped around the $\mathrm{Ti}$ wire were connected to external circuit with silver paste for subsequent structure and microsupercapacitor performance characterization.

Fig. 2a shows a typical SEM image of an as-prepared anodized titanium wire, which shows a relatively rough surface compared with the pristine wire before anodization. SEM images of the anodized titanium wire under higher magnifications were given in Figs. 2b, c, which clearly show the aligned titania nanotubes with a tube length of about $10 \mu \mathrm{m}$ (Fig. S1). The X-ray Diffraction (XRD) spectra in Fig. S2 
confirm the presence of $\mathrm{TiO}_{2}$ outside of the $\mathrm{Ti}$ wire upon anodization, and a highly crystalline anatase phase for the $\mathrm{TiO}_{2}$ nanotube after annealing. As mentioned above, the aligned CNT sheet and yarn were prepared through a dry-spinning process [13] from a spinnable vertically-aligned CNT array (Figs. 2d, e and Fig. S3).[27-34] Figs. $\mathrm{S} 4 \mathrm{a}, \mathrm{b}$ show SEM images of the CNT spun fiber with a diameter of $18 \mu \mathrm{m}$ and a uniform morphology consisting of nanotubes aligned with each other within the yarn. The corresponding Raman spectrum in Fig. S5 shows a G-band peak at $1574 \mathrm{~cm}^{-1}$ and a D-band peak at $1344 \mathrm{~cm}^{-1}$ with a high peak intensity ratio of $\mathrm{I}_{\mathrm{G}} / \mathrm{I}_{\mathrm{D}}(1.16)$, indicating a high quality and low defct content for the CNTs. The high-quality, highly aligned CNT structure can efficiently enhance charge transport through the CNT yarn, which is attractive for device applications. As seen in Figs. 2d, e, a highly aligned CNT sheet can be continuously drawn from the spinnable vertically-aligned CNT array and be in-situ wrapped around the $\mathrm{Ti}$ wire for the construction of wire-shaped microsupercapacitors.

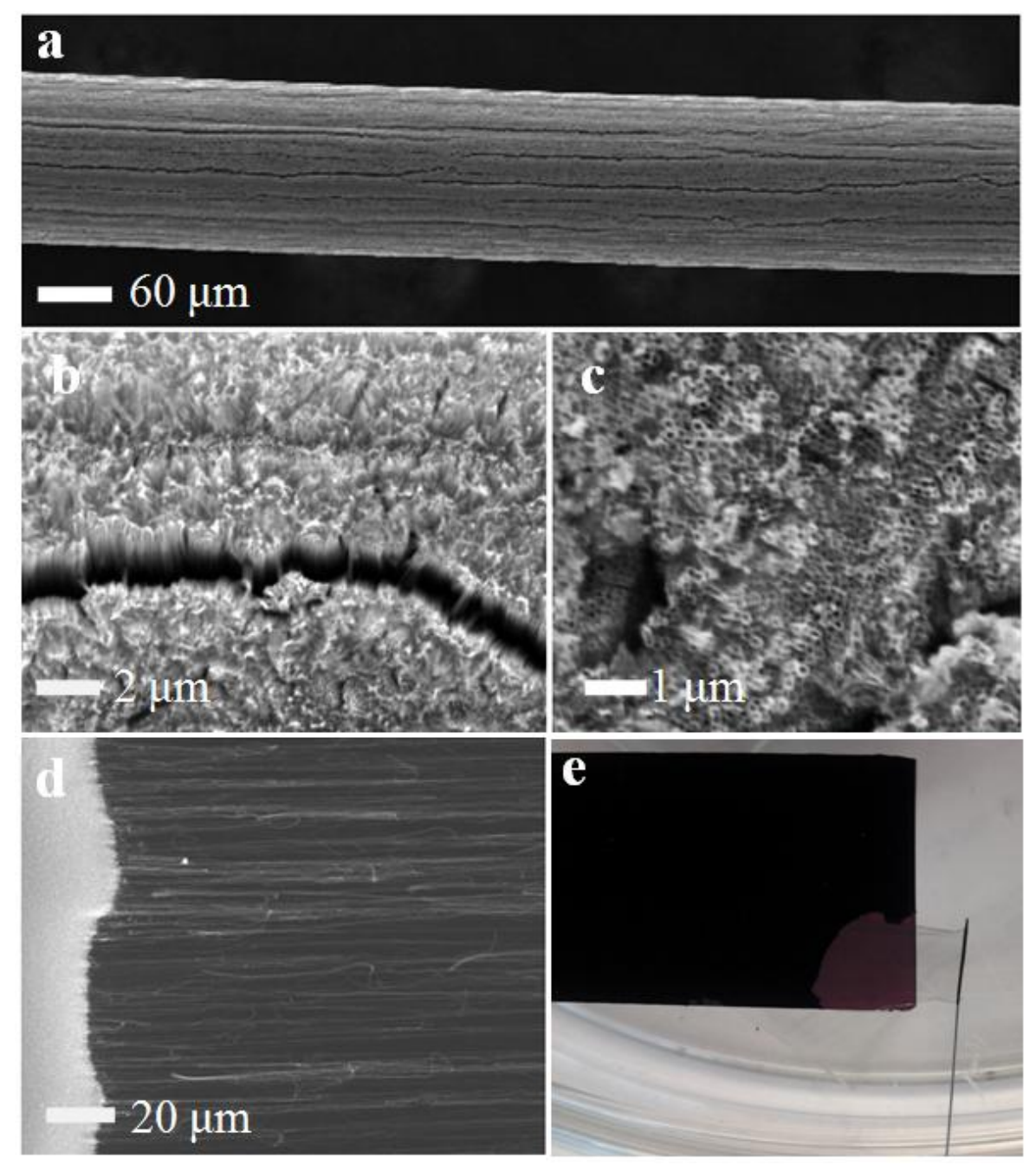

Fig. 2. Morphology of the anodized Ti wire and aligned CNTs. (a) SEM image of an 
anodized Ti wire. (b, c) SEM images of the radially aligned titania nanotubes around the Ti wire. (d) SEM image of aligned CNT sheet drawing from the vertically-aligned CNT array. (e) Optical photograph of the process for drawing the CNT sheet from the vertically-aligned CNT array, followed by in-situ wrapping around an anodized Ti wire.

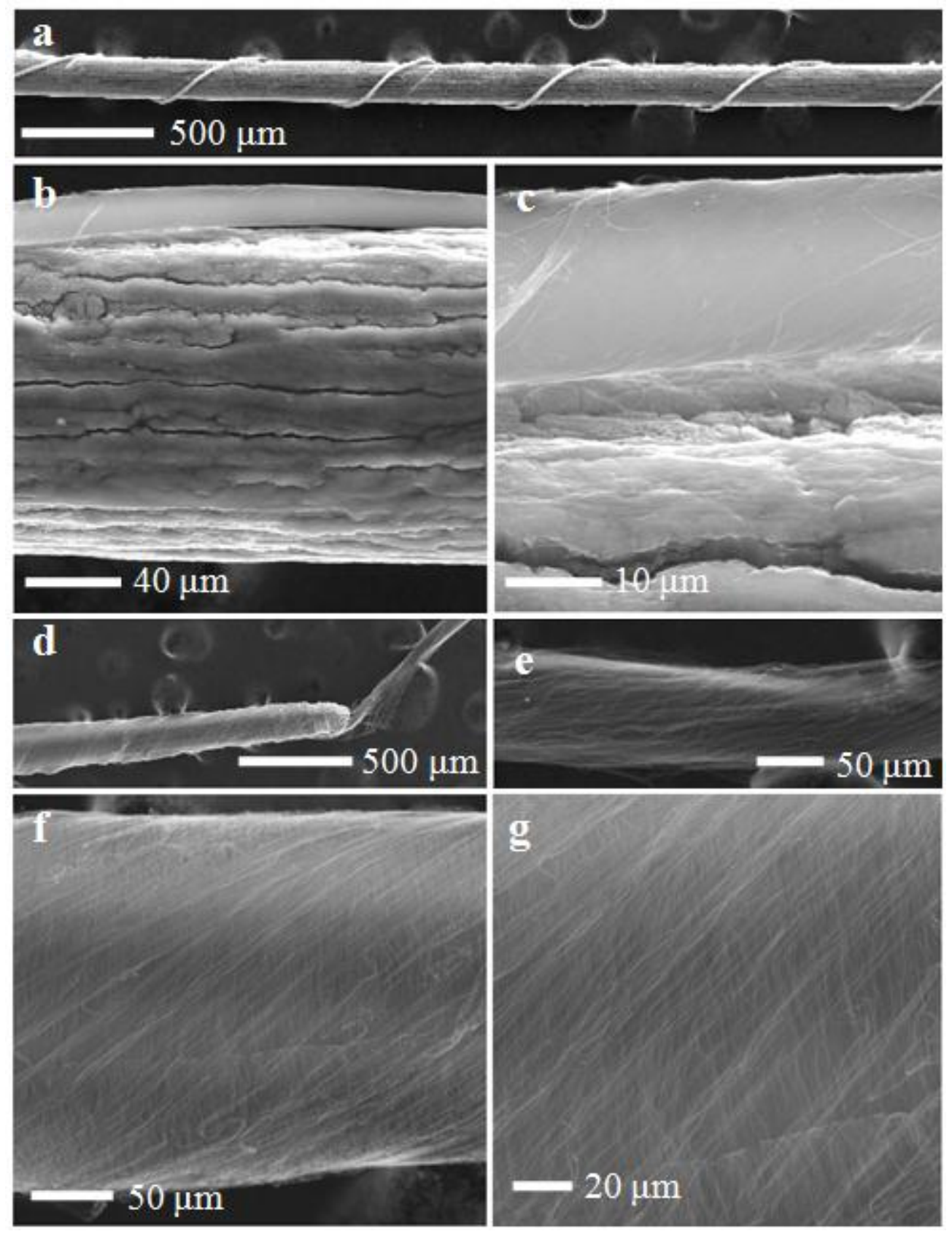

Fig. 3. Morphology of the wire-shaped microsupercapacitors. (a, b) SEM images of a wire-shaped microsupercapacitor using a single CNT yarn wrapped the titania nanotubes supported by a titanium wire as one of the electrodes under low (a) and high (b) magnifications. (c) SEM image of the interface between the titania nanotubes and CNT yarn. (d) SEM image of a microsupercapacitor using aligned CNT sheet 
wrapped the titania nanotubes supported by a titanium wire as the second electrode. (e) SEM image of the aligned CNT bundle formed from the CNT sheet at the end of the microsupercapacitor for connecting to an external circuit. (f, g) Low and high magnification SEM images of a Ti wire wrapped with an aligned and continuous CNT sheet.

The wire-shaped microsupercapacitors were fabricated by twisting aligned CNT yarns as the second electrode around the Ti wire sheathed with radially aligned titania nanotubes as the primary electrode. Figs. 3a, b show SEM images of the microsupercapacitor thus prepared. Due to its high flexibility and tensile strength, the CNT yarn can be readily twisted around the Ti wire with an intimated contact for efficient charge transport (Fig. 3c). However, limited area of the Ti wire was covered when only a single CNT yarn was used, which could lead to a poor device performance. Therefore, microsupercapacitors with similar structures using the titanium wire sheathed with radially aligned titania nanotubes wrapped by two or three CNT yarns were constructed (Figure S6). As expected, devices using two or three CNT yarns showed a much better performance than their counterparts based on a single CNT yarn (vide infra), though still a large portion of the Ti wire did not contribute to the device performance. As an alternative, therefore, microsupercapacitors with a large-area aligned CNT sheet directly wrapped around the Ti wire continuously were fabricated (Figs. 2e, 3d). Fig. 3d shows an SEM image of one end for such a device with the CNT sheet bundled together for connecting to an external circuit (Fig. 3e). Figs. 3f, g show that CNTs highly aligned with each other and fully covered the outside surface of the $\mathrm{Ti}$ wire, leading to efficient charge transport for high device performance (vide infra).

To evaluate the electrochemical performance of the wire-shaped microsupercapacitors, we performed cyclic voltammetry (CV), galvanostatic charge/discharge, and electrochemical impedance spectroscopic (EIS) measurements. Fig. 4a shows CV curves of the microsupercapacitors using CNT yarns or sheet as the second electrode at a scan rate of $0.1 \mathrm{~V} \mathrm{~s}^{-1}$. The rectangular shape of the obtained CV curves is an indication for the electrical double layer capacitance. Typical galvanostatic charge/discharge curves of the wire-shaped microsupercapacitors were given in Fig. 4b, showing the symmetric triangular shape for a nearly ideal capacitive behavior. The specific capacitance can be calculated using the equation of $C_{s p}=$ $2 I \Delta t / S \Delta V$, where $I$ is the applied current, $\Delta t$ is the discharging time, and $\Delta V$ is the voltage window, respectively. Here, $S$ is the surface area of a fiber-shaped 
supercapacitor, which was calculated by multiplying the circumference of the Ti wire and the effective device length. As seen in Figs. 4a, c, the specific capacitance (per electrode area) of the wire-shaped microsupercapacitor increased from 0.57 through 0.90 to $1.04 \mathrm{mF} \mathrm{cm}^{-2}$ as the number of the CNT yarn increased from one through two to three. The capacitance of the microsupercapacitor with a CNT sheet electrode reached to $1.84 \mathrm{mF} \mathrm{cm}^{-2}$ that was about three time of that for the device based on a single CNT yarn electrode, attributable to the increased effective area of the electrode. The performance of this newly-developed wire-shaped microsupercapacitor is comparable with that of the CNT fiber-based device using liquid electrolyte.[13] The specific volumetric capacitance of the CNT sheet-based microsupercapacitor is about $1.84 \mathrm{mF} \mathrm{cm}^{-3}$, and corresponding energy density and power density are $0.16 \times 10^{-3}$ $\mathrm{mWh} \mathrm{cm}{ }^{-3}$ and $0.01 \mathrm{~mW} \mathrm{~cm}^{-3}$, respectively. The dependence of specific capacitance on current is given in Fig. 4c, which shows that the specific capacitance of the microsupercapacitors with CNT yarns reduced with increasing current much faster than that of devices based on the CNT sheet. Thus, the wire-shaped microsupercapacitor with the CNT sheet electrode exhibited a higher stability than its counterparts based on the CNT yarns. The capacitance for both type devices retained at least $80 \%$ (Fig. S7) even after 1000 charge-discharge cycles, suggesting reasonably good stabilities. Nyquist plots measured over a frequency range from $10^{-2}$ to $10^{5} \mathrm{~Hz}$ are given in Fig. 4d, showing the vertical lines at low frequencies characteristic of a pure capacitive behavior. The series resistances of microsupercapacitors based on the CNT yarns decreased from 580 through 240 to $160 \Omega$ as the number of the CNT yarns increased from one through two to three, in a good consistence with the improved device performance as the number of CNT yarns increased. 

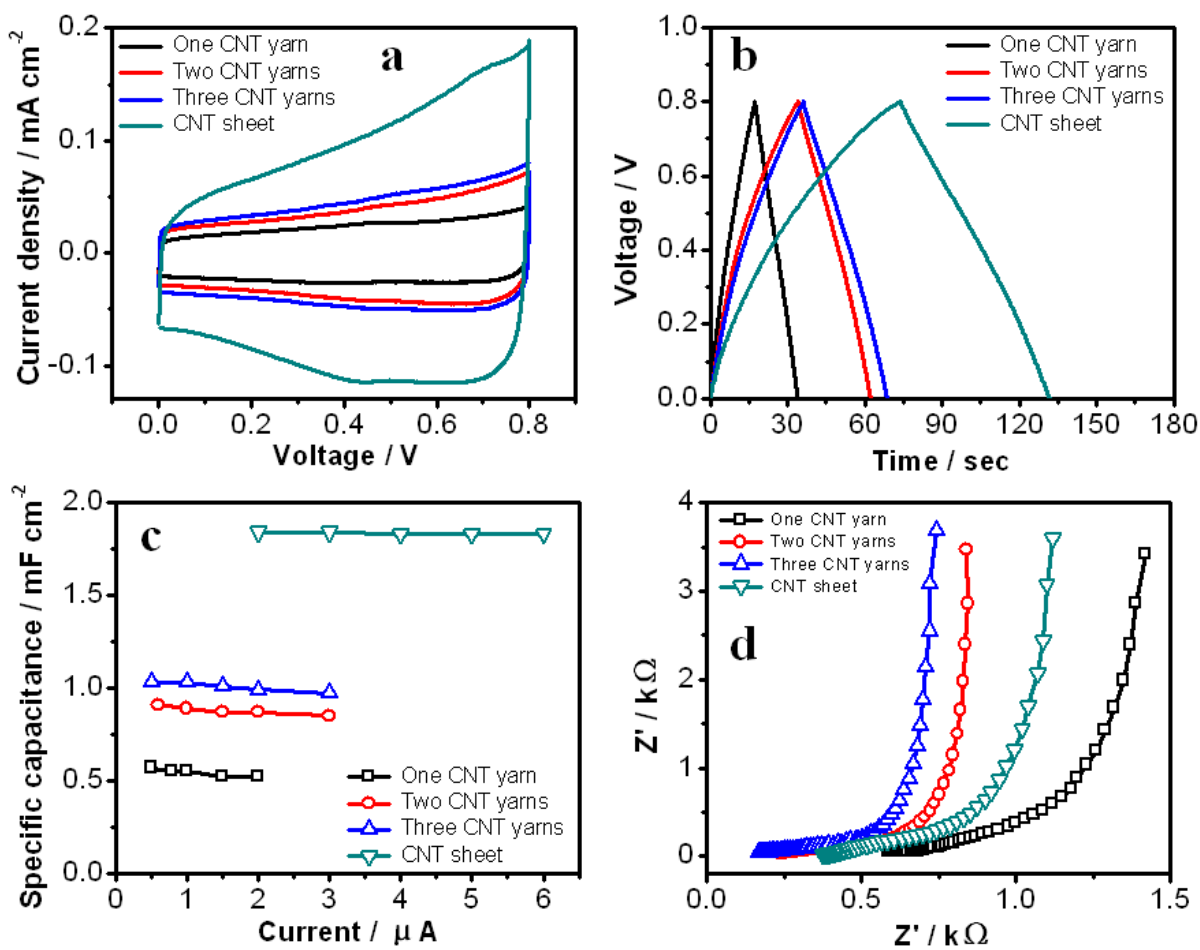

Fig. 4. Electrochemical performance of the wire-shaped microsupercapacitors with the CNT yarns or sheet electrodes. (a) CV curves measured at a scan rate of $0.1 \mathrm{~V} \mathrm{~s}^{-1}$. (b) The galvanostatic charging-discharging curves of the microsupercapacitors at a constant current of $1 \mu \mathrm{A}$. (c) The dependence of specific capacitance of various devices on the current. (d) Nyquist plots of the microsupercapacitors within the frequency range from $10^{-2}$ to $10^{5} \mathrm{~Hz}$. 

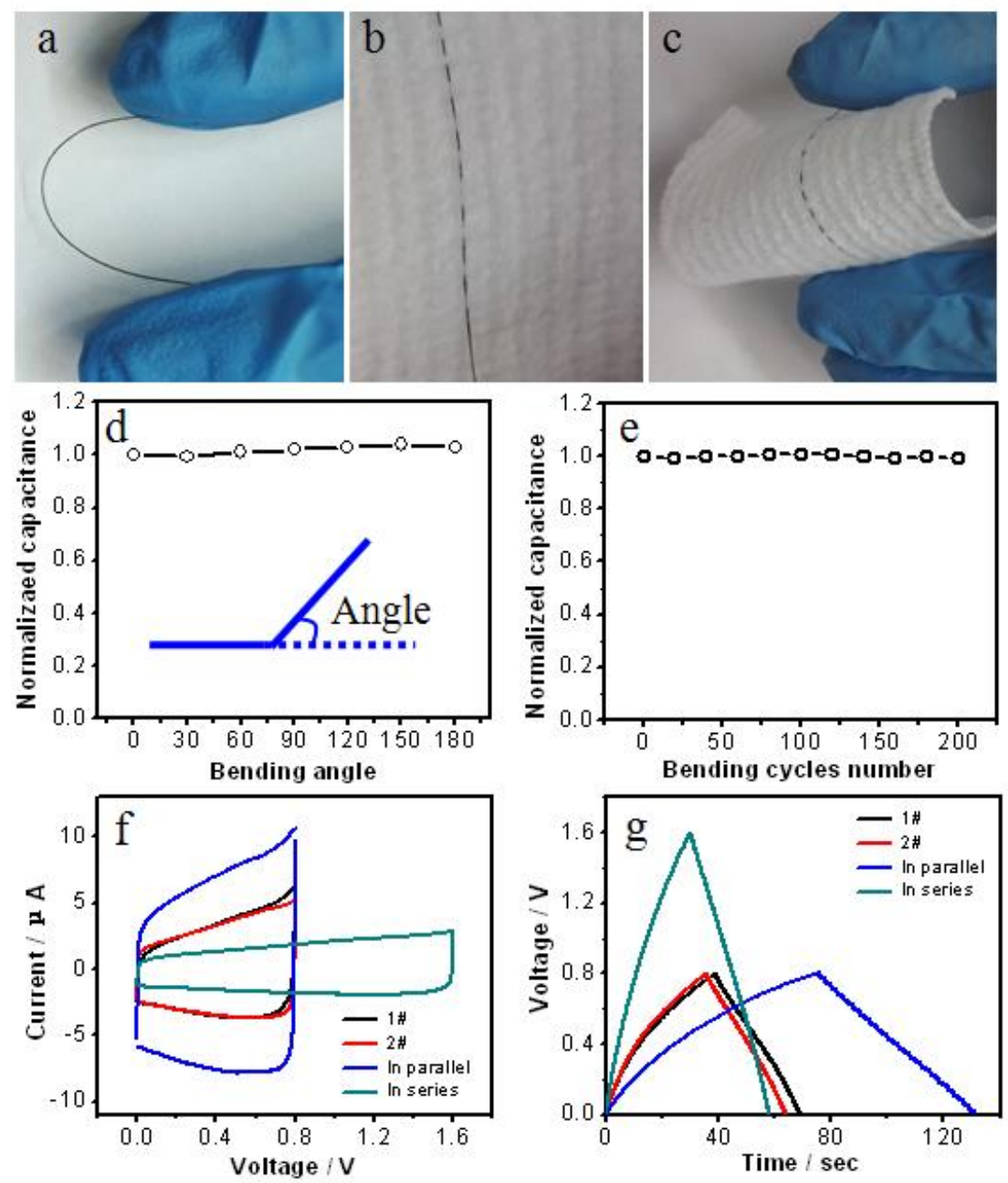

Fig. 5. Flexibility and properties of the wire-shaped microsupercapacitors, these devices were fabricated using the second electrode with two CNT yarns. (a) Optical photograph of a wire-shaped device at a bending state. (b, c) Optical photographs of the wire-shaped microsupercapacitor woven into a white textile without (b) and with bending (c). (d) Dependence of the specific capacitance on the bending angles. (e) Dependence of the specific capacitance on the bending cycles. (f) $\mathrm{CV}$ curves of two microsupercapacitors in series and parallel at a scan rate of $0.1 \mathrm{~V} \mathrm{~s}^{-1}$. (g) The galvanostatic charging-discharging curves of two microsupercapacitors in series and parallel at a constant current of $1 \mu \mathrm{A}$.

As expected, these wire-shaped microsupercapacitors are so flexible that they can be bent over a large angle (Fig. 5a) and woven into a textile (Figs. 5b, c). Fig. S8 shows CV and charge-discharge curves of a typical microsupercapacitor when it was bent to different angles ranging from 0 to 180 degree (Fig. 5d), indicating a good flexibility and stability. Besides, these wire-shaped microsupercapacitors can be bent 
for hundreds of times without any obvious change in electrochemical performance (Figs. 5e, S9). Furthermore, the output current and voltage of these wire-shaped microsupercapacitors can be easily tuned by connecting them in series or in parallel (Figs. 5f, g). For instance, an output voltage of $1.6 \mathrm{~V}$ could be achieved by connecting two microsupercapacitors in series, and the output current can be tuned up to the sum of that of the two devices by connecting them in parallel.

\section{Conclusions}

In summary, we have developed a series of wire-shaped microsupercapacitors by using highly aligned CNT yarn or sheet as the electrode, which was wrapped around a Ti wire sheathed with radially aligned titania nanotubes as the other electrode. Our results demonstrated that the specific capacitance of the microsupercapacitors based on the CNT yarn electrode increased as the number of the CNT yarn increased, and the devices using the CNT sheet electrode showed the highest capacitance $(1.84 \mathrm{mF}$ $\mathrm{cm}^{-2}$ ) due to its large effective area. The wire-shaped microsupercapacitors are highly flexible; their capacitance almost unchanged when they were bent at different angles ranging from 0 to 180 degree. The wire-shaped microsupercapacitors also showed good flexibility and stabilities with almost no change in electrochemical performance over hundreds bending cycles. Furthermore, the output voltage and current can be easily tuned by integrating multiple of the microsupercapacitors in series and in parallel, respectively. These newly-developed flexible and wearable energy storage devices show great potential to be used as energy sources for portable and/or wearable electronics and many other applications.

\section{Acknowledgment}

The authors acknowledge support of NSF (CMMI-1400274, CMMI-1266295), DOD-AFOSR-MURI (FA9550-12-1-0037), and AFRL/DAGSI (RQ20-CWRU-13-4). 


\section{Reference}

[1] B. Weintraub, Y. Wei, Z. L. Wang, Angew. Chem. Int. Ed. 48 (2009) 8981.

[2] M. R. Lee, R. D. Eckert, K. Forberich, G. Dennler, C. J. Brabec, R. A. Gaudiana, Science 324 (2009) 232.

[3] T. Chen, L. Qiu, Z. Yang, H. Peng, Chem. Soc. Rev. 42 (2013) 5031.

[4] X. Fan, Z. Z. Chu, F. Z. Wang, C. Zhang, L. Chen, Y. W. Tang, D. C. Zou, Adv. Mater. 20 (2008) 592.

[5] Y. P. Fu, Z. B. Lv, S. C. Hou, H. W. Wu, D. Wang, C. Zhang, Z. Z. Chu, X. Cai, X. Fan, Z. L. Wang, D. C. Zou, Energy Environ. Sci. 4 (2011) 3379.

[6] Y. Qin, X. D. Wang, Z. L. Wang, Nature 451 (2008) 809.

[7] A. Yadav, K. P. Pipe, M. Shtein, J. Power Sources 175 (2008) 909.

[8] X. Xiao, T. Li, P. Yang, Y. Gao, H. Jin, W. Ni, W. Zhan, X. Zhang, Y. Cao, J. Zhong, L. Gong, W.-C. Yen, W. Mai, J. Chen, K. Huo, Y.-L. Chueh, Z. L. Wang, J. Zhou, ACS Nano 6 (2012) 9200.

[9] K. Wang, Q. Meng, Y. Zhang, Z. Wei, M. Miao, Adv. Mater. 25 (2013) 1494.

[10]X. Li, X. Zang, Z. Li, X. Li, P. Li, P. Sun, X. Lee, R. Zhang, Z. Huang, K. Wang, D. Wu, F. Kang, H. Zhu, Adv. Funct. Mater. 23 (2013) 4862.

[11]B. Liu, D. Tan, X. Wang, D. Chen, G. Shen, Small 9 (2013) 1998.

[12]Z. Cai, L. Li, J. Ren, L. Qiu, H. Lin, H. Peng, J. Mater. Chem. A, 2013, 1, 258.

[13]J. Ren, L. Li, C. Chen, X. Chen, Z. Cai, L. Qiu, Y. Wang, X. Zhu, H. Peng, Adv. Mater. 25 (2013) 1155.

[14]P. Simon, Y. Gogotsi, Nat. Mater. 7 (2008) 845.

[15]S. H. Mujawar, S. B. Ambade, T. Battumur, R. B. Ambade, S.-H. Lee, Electrochimica Acta 56 (2011) 4462.

[16]C. Xiang, M. Li, M. Zhi, A. Manivannanc, N. Wu, J. Mater. Chem. 22 (2012) 19161.

[17]F. Gobal, M. Faraji, J. Electroanal. Chem. 691 (2013) 51.

[18]S. Bose, T. Kuila, A. K. Mishra, R. Rajasekar, N. H. Kim J. H. Lee, J. Mater. Chem. 22 (2012) 767.

[19]L. Dai, D. W. Chang, J.-B. Baek, W. Lu, Small 8 (2012) 1130.

[20]D. N. Futaba, K. Hata, T. Yamada, T. Hiraoka, Y. Hayamizu, Y. Kakudate, O. Tanaike, H. Hatori, M. Yumura, S. Iijima, Nat. Mater. 5 (2006) 987.

[21]Y. Zhu, S. Murali, M. D. Stoller, K. J. Ganesh, W. Cai, P. J. Ferreira, A. Pirkle, R. M. Wallace, K. A. Cychosz, M. Thommes, D. Su, E. A. Stach, R. S. Ruoff, Science 332 (2011) 1537. 
[22]Z. S. Wu, K. Parvez, X. L. Feng, K. Müllen, Nat. Commun. 4 (2013) 2487.

[23]Z. S. Wu, K. Parvez, A. Winter, H. Vieker, X. Liu, S. Han, A. Turchanin, X. Feng, K. Müllen, Adv. Mater. 26 (2014) 4552.

[24]Z. S. Wu, K. Parvez, S. Li, S. Yang, Z. Y. Liu, S. H. Liu, X. L. Feng, K. Müllen, Adv. Mater. 27 (2015) 4054.

[25]Z. S. Wu, K. Parvez, X. L. Feng, K. Müllen, J. Mater. Chem. A 2 (2014) 8288.

[26]Z. S. Wu, X. L. Feng, H. M. Cheng. Natl. Sci. Rev. 1 (2014) 277.

[27]H. Peng, X. Sun, F. Cai, X. Chen, Y. Zhu, G. Liao, D. Chen, Q. Li, Y. Lu, Y. Zhu Q. Jia, Nat Nanotechnol. 4 (2009) 738.

[28]H. Peng, M. Jain, Q. Li, D. E. Peterson, Y. Zhu Q. Jia, J. Am. Chem. Soc. 130 (2008) 1130.

[29]T. Chen, L. Qiu, Z. Yang, H. G. Kia, H. Peng. Adv. Mater. 24 (2012) 4623.

[30] K. Jiang, J. Wang, Q. Li, L. Liu, C. Liu S. Fan, Adv. Mater. 23 (2011) 1154.

[31]K. Koziol, J. Vilatela, A. Moisala, M. Motta, P. Cunniff, M. Sennett A. Windle, Science 318 (2007) 1892.

[32] Y.-L. Li, I. A. Kinloch A. H. Windle, Science 304 (2004) 276.

[33]M. Zhang, K. R. Atkinson R. H. Baughman, Science 306 (2004) 1358.

[34]B. Vigolo, A. Pénicaud, C. Coulon, C. Sauder, R. Pailler, C. Journet, P. Bernier P. Poulin, Science 290 (2000) 1331. 
Table of Contents

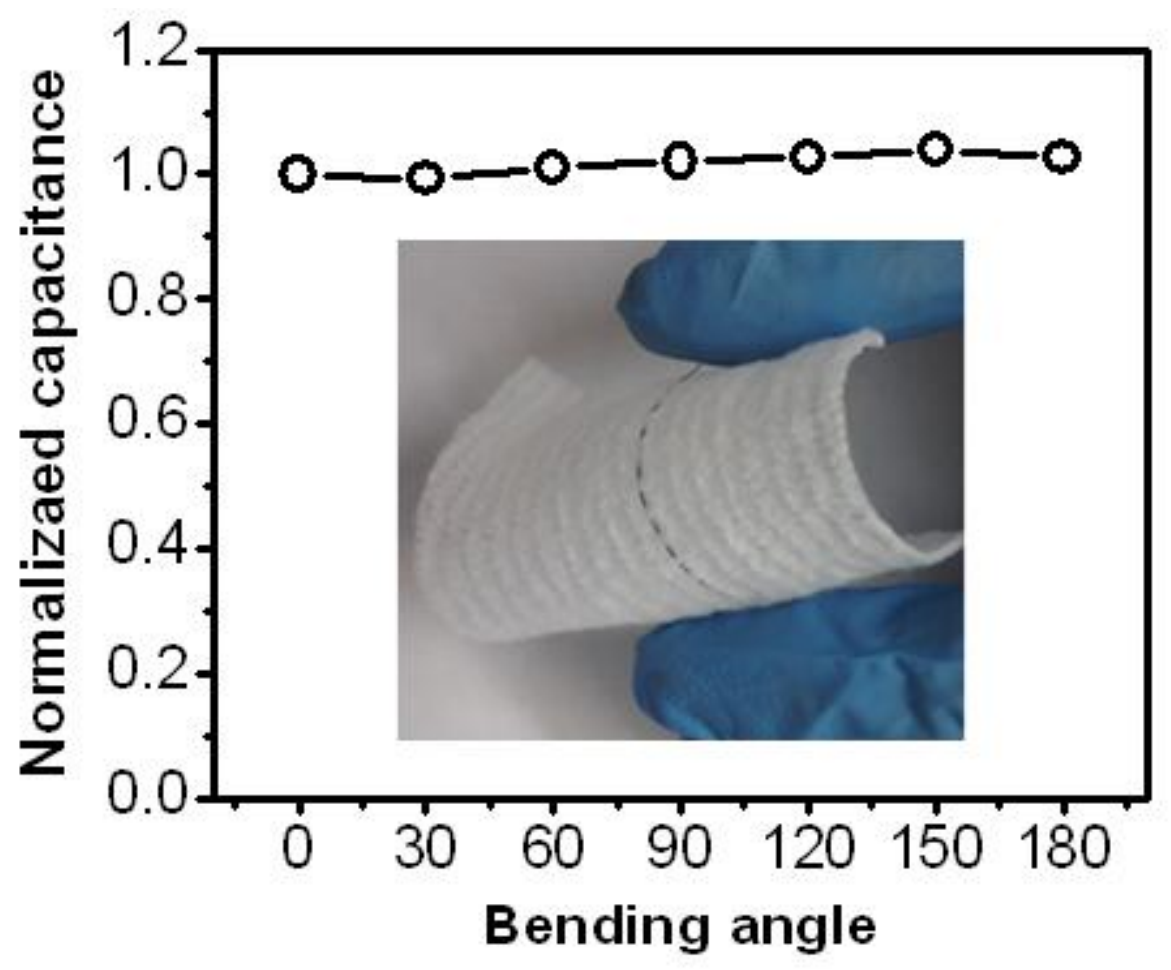

